\title{
ON SCHUR'S SECOND PARTITION THEOREM
}

\author{
by GEORGE E. ANDREWS $\dagger$
}

(Received 20 December, 1966)

1. Introduction. In 1926, I. J. Schur proved the following theorem on partitions [3].

THEOREM 1. The number of partitions of $n$ into parts congruent to $\pm 1(\bmod 6)$ is equal to the number of partitions of $n$ of the form $b_{1}+\ldots+b_{s}=n$, where $b_{i}-b_{i+1} \geqq 3$ and, if $3 \mid b_{i}$, then $b_{i}-b_{i+1}>3$.

Schur's proof was based on a lemma concerning recurrence relations for certain polynomials. In 1928, W. Gleissberg gave an arithmetic proof of a strengthened form of Schur's theorem [2]; however, the combinatorial reasoning in Gleissberg's paper becomes very intricate.

Although claims of simplicity of proof are highly subjective, we shall in $\$ 2$ give a proof of Schur's theorem which is shorter than the two previous proofs and seems to exhibit the crucial steps more clearly. This new proof depends on Appell's Comparison Theorem $[1$, p. 101]. In $\S 3$, we generalize our technique and prove a new partition theorem of which the following is a special case.

THEOREM 4. Let $A(n)$ denote the number of partitions of $n$ into parts congruent to $0,2,3$, $4,7(\bmod 8)$. Let $B(n)$ denote the number of partitions of $n$ of the form $n=b_{1}+\ldots+b_{s}$, where $b_{s} \geqq 2, b_{i} \geqq b_{i+1}$, and, if $b_{i}$ is odd, $b_{i}-b_{i+1} \geqq 3$. Then $A(n)=B(n)$.

For example, if $n=15$, the nineteen partitions enumerated by $A(15)$ are $15,12+3$, $11+4,11+2+2,10+3+2,8+7,8+4+3,8+3+2+2,7+4+4,7+4+2+2,7+3+3+2$, $7+2+2+2+2, \quad 4+4+4+3, \quad 4+4+3+2+2, \quad 4+3+3+3+2, \quad 4+3+2+2+2+2$, $3+3+3+3+3,3+3+3+2+2+2,3+2+2+2+2+2+2$. The nineteen partitions enumerated by $B(15)$ are $15,13+2,12+3,11+4,11+2+2,10+5,10+3+2,9+6,9+4+2,9+2+$ $2+2,8+7,8+5+2,8+4+3,7+4+4,7+4+2+2,7+2+2+2+2,6+6+3,6+5+2+2$, $4+4+4+3$.

Finally in \$4, we show how Schur's lemma concerning recurrence relations for certain polynomials is actually a direct corollary of the $q$-analogue of Gauss's theorem for hypergeometric series.

2. Proof of Theorem 1. Let $\pi(n)$ denote the number of partitions of $n$ of the form $n=b_{1}+\ldots+b_{s}$ with $b_{i}-b_{i+1} \geqq 3$ and $b_{i}-b_{i+1}>3$ if $3 \mid b_{i}$. Let $\pi_{m}(n)$ denote the number of partitions just described, with the added condition that $b_{1} \leqq m$. By breaking the set of partitions enumerated by $\pi_{m}(n)$ into two sets, those with largest part less than $m$ and those with largest part equal to $m$, we see that

$$
\begin{aligned}
& \pi_{3 m+1}(n)=\pi_{3 m}(n)+\pi_{3 m-2}(n-3 m-1), \\
& \pi_{3 m+2}(n)=\pi_{3 m+1}(n)+\pi_{3 m-1}(n-3 m-2), \\
& \pi_{3 m+3}(n)=\pi_{3 m+2}(n)+\pi_{3 m-1}(n-3 m-3) .
\end{aligned}
$$

$\dagger$ Partially supported by National Science Foundation Grant GP-5593. 
If

and

$$
d_{m}(q)=1+\sum_{n=1}^{\infty} \pi_{m}(n) q^{n}
$$

$$
d(q)=1+\sum_{n=1}^{\infty} \pi(n) q^{n}
$$

then for $|q|<1, d_{m}(q) \rightarrow d(q)$ as $m \rightarrow \infty$, since

$$
\left|d(q)-d_{m}(q)\right| \leqq \sum_{n=m}^{\infty} p(n)|q|^{n}
$$

where $p(n)$ is the ordinary partition function. From (2.1), (2.2) and (2.3) we deduce

$$
\begin{aligned}
& d_{3 m+1}(q)=d_{3 m}(q)+q^{3 m+1} d_{3 m-2}(q), \\
& d_{3 m+2}(q)=d_{3 m+1}(q)+q^{3 m+2} d_{3 m-1}(q), \\
& d_{3 m+3}(q)=d_{3 m+2}(q)+q^{3 m+3} d_{3 m-1}(q) .
\end{aligned}
$$

Let

$$
\alpha_{m}(q)=d_{3 m+2}(q)
$$

Then, by (2.6),

By (2.5),

$$
d_{3 m+3}(q)=\alpha_{m}(q)+q^{3 m+3} \alpha_{m-1}(q) .
$$

$$
d_{3 m+1}(q)=\alpha_{m}(q)-q^{3 m+2} \alpha_{m-1}(q) .
$$

Hence, by substituting (2.7) and (2.8) into (2.4), we obtain

$$
\alpha_{m}(q)=\left(1+q^{3 m+1}+q^{3 m+2}\right) \alpha_{m-1}(q)+q^{3 m}\left(1-q^{3 m}\right) \alpha_{m-2}(q) .
$$

We note that $\alpha_{m}(q)$ is uniquely determined by (2.9) and the two initial values $\alpha_{-1}(q)=1$, $\alpha_{0}(q)=1+q+q^{2}$.

Now, for $|x|<1,|q|<1$, define $s_{n}(q)$ by

and let

$$
\prod_{n=0}^{\infty}\left(1+x q^{3 n+1}\right)\left(1+x q^{3 n+2}\right)\left(1-x q^{3 n}\right)^{-1}=\sum_{n=0}^{\infty} s_{n}(q) x^{n}
$$

$$
S_{n}(q)=\prod_{j=1}^{n}\left(1-q^{3 j}\right) \cdot s_{n}(q)
$$

Calling the expression on the left-hand side of $(2.10) f(x ; q)$, we have

$$
(1-x) f(x ; q)=(1+x q)\left(1+x q^{2}\right) f\left(x q^{3} ; q\right) .
$$

Hence $s_{0}(q)=1, s_{1}(q)=(1-q)^{-1}$ and, for $n>1$,

$$
s_{n}(q)-s_{n-1}(q)=q^{3 n} s_{n}(q)+q^{3 n-2} s_{n-1}(q)+q^{3 n-1} s_{n-1}(q)+q^{3 n-3} s_{n-2}(q) .
$$


Thus

Therefore

$$
\left(1-q^{3 n}\right) s_{n}(q)=\left(1+q^{3 n-2}+q^{3 n-1}\right) s_{n-1}(q)+q^{3 n-3} s_{n-2}(q) .
$$

and

$$
S_{n}(q)=\left(1+q^{3 n-2}+q^{3 n-1}\right) S_{n-1}(q)+q^{3 n-3}\left(1-q^{3 n-3}\right) S_{n-2}(q),
$$

$$
S_{0}(q)=1, \quad S_{1}(q)=1+q+q^{2} .
$$

Hence, by the remarks following (2.6), $S_{n+1}(q)=\alpha_{n}(q)$.

Thus, for $|x|<1,|q|<1$,

$$
\prod_{n=0}^{\infty}\left(1+x q^{3 n+1}\right)\left(1+x q^{3 n+2}\right)\left(1-x q^{3 n}\right)^{-1}=\sum_{m=0}^{\infty}\left(\alpha_{m-1}(q) x^{m} / \prod_{j=1}^{m}\left(1-q^{3 j}\right)\right) .
$$

Hence, by Appell's comparison theorem $\left[1\right.$, p. 101, with $\left.p_{n}=1\right]$,

$$
\begin{aligned}
\prod_{n=0}^{\infty}\left(1+q^{3 n-1}\right)\left(1+q^{3 n+2}\right)\left(1-q^{3 n+3}\right)^{-1} & =\lim _{x \rightarrow 1}(1-x) \sum_{m=0}^{\infty}\left(\alpha_{m-1}(q) x^{m} / \prod_{j=1}^{m}\left(1-q^{3 j}\right)\right) \\
& =\lim _{m \rightarrow \infty} \alpha_{m-1}(q) \prod_{j=1}^{m}\left(1-q^{3 j}\right)^{-1} \\
& =d(q) \prod_{n=1}^{\infty}\left(1-q^{3 n}\right)^{-1} .
\end{aligned}
$$

Hence

$$
\begin{aligned}
d(q) & =\prod_{n=0}^{\infty}\left(1+q^{3 n+1}\right)\left(1+q^{3 n+2}\right) \\
& =\prod_{n=0}^{\infty}\left(1-q^{6 n+1}\right)^{-1}\left(1-q^{6 n+5}\right)^{-1} .
\end{aligned}
$$

Consequently, comparing coefficients of $q^{N}$ on both sides of $(2.13)$, we see that $\pi(N)$ is also the number of partitions of $N$ into parts congruent to $\pm 1(\bmod 6)$.

A slight refinement of the above argument will yield Gleissberg's generalization of Schur's theorem [2, p. 374].

3. Generalizations. We may extend our previous argument to prove the following theorem.

THEOREM 2. Let $q$ be real with $0<q<1$, and $a_{i} \geqq 0$ for $1 \leqq i \leqq r$. If $t_{0}=1, t_{n}=0$ for $n<0$, and for $n>0$

then

$$
t_{n}=\left(1+a_{1} q^{n}\right) t_{n-1}+\sum_{j=2}^{r} a_{j} q^{n} t_{n-j} \prod_{s=1}^{j-1}\left(1-q^{n-s}\right)
$$

$$
\lim _{n \rightarrow \infty} t_{n}=\prod_{m=1}^{\infty}\left(1+a_{1} q^{m}+a_{2} q^{2 m}+\ldots+a_{r} q^{r m}\right)
$$


Proof. If here we let

$$
f_{r}(x ; q)=\prod_{m=1}^{\infty}\left(1+a_{1} x q^{m}+a_{2} x q^{2 m}+\ldots+a_{r} x q^{r m}\right)\left(1-x q^{m-1}\right)^{-1},
$$

and write

$$
f_{r}(x ; q)=\sum_{n=0}^{\infty} \beta_{n}(q) x^{n}
$$

then from

$$
(1-x) f_{r}(x ; q)=\left(1+a_{1} x q+a_{2} x q^{2}+\ldots+a_{r} x q^{r}\right) f_{r}(x q ; q)
$$

we deduce that

$$
t_{n}=\beta_{n}(q) \prod_{j=1}^{n}\left(1-q^{j}\right)
$$

Now $t_{0}=1>0$. Suppose that, for $0 \leqq n<m, t_{n}>0$; then

$$
\begin{aligned}
t_{m}-t_{m-1} & =a_{1} q^{m} t_{m-1}+\sum_{j=2}^{r} a_{j} q^{m} t_{m-j} \prod_{s=1}^{j-1}\left(1-q^{m-s}\right) \\
& \geqq 0
\end{aligned}
$$

Thus, by mathematical induction, $t_{m}(m>0)$ is a non-decreasing sequence of positive numbers.

Consequently,

Hence, for all $m \geqq 0$,

$$
\begin{aligned}
t_{m} & \leqq\left(1+a_{1} q^{m}\right) t_{m-1}+\sum_{j=2}^{r} a_{j} q^{m} t_{m-1} \\
& =\left(1+\left(a_{1}+\ldots+a_{r}\right) q^{m}\right) t_{m-1}
\end{aligned}
$$

$$
t_{m} \leqq \prod_{n=0}^{\infty}\left(1+\left(a_{1}+\ldots+a_{r}\right) q^{n}\right)
$$

Thus $t_{m}$ is a non-decreasing bounded sequence of positive terms, and therefore $t_{m}$ converges to a limit $L$.

Hence, by Appell's comparison theorem $\left[1\right.$, p. 101 with $\left.p_{n}=1\right]$, we deduce as in Theorem 1 that

$$
\lim _{n \rightarrow 0} t_{n}=L=\prod_{m=1}^{\infty}\left(1+a_{1} q^{m}+a_{2} q^{2 m}+\ldots+a_{r} q^{r m}\right) .
$$

Thus Theorem 2 is proved.

As an example of Theorem 2, we prove the following partition theorem.

THEOREM 3. Let $r \geqq 2$ be an integer. Let $P_{1}(n)$ denote the number of partitions of $n$ into parts which are either even and not congruent to $4 r-2(\bmod 4 r)$ or odd and congruent to $2 r-1,4 r-1(\bmod 4 r)$. Let $P_{2}(n)$ denote the number of partitions of $n$ of the form $n=b_{1}+\ldots$ $+b_{s}$, where $b_{i} \geqq b_{i+1}$, and for $b_{i}$ odd, $b_{i}-b_{i+1} \geqq 2 r-1\left(1 \leqq i \leqq s\right.$, where $\left.b_{s+1}=0\right)$. Then $P_{1}(n)=P_{2}(n)$. 
Proof. Let $p(n, m)$ denote the number of partitions of $n$ of the type enumerated by $P_{2}(n)$, with the added restriction that $b_{1} \leqq 2 m$. Let

First we shall prove that

$$
B_{m}(q)=1+\sum_{n=1}^{\infty} p(n, m) q^{n} .
$$

$$
p(n, m)-p(n, m-1)=p(n-2 m, m)+p(n-2 m+1, m-r) .
$$

Now $p(n, m)-p(n, m-1)$ denotes the number of partitions of the type enumerated by $p(n, m)$ with the added restriction that either $2 m$ or $2 m-1$ is the largest part. If $2 m$ is the largest part, remove it. This yields a partition of the type enumerated by $p(n-2 m, m)$. If $2 m-1$ is the largest part, then the next largest part does not exceed $2 m-2 r$. Hence, if $2 m-1$ is removed from the partition under consideration, we obtain a partition of the type enumerated by $p(n-2 m+1, m-r)$. Thus the above procedure establishes a one-to-one correspondence between those partitions enumerated by $p(n, m)-p(n, m-1)$ and the totality of partitions which are enumerated either by $p(n-2 m, m)$ or by $p(n-2 m+1, m-r)$. Thus (3.10) is established.

Equation (3.10) implies that

$$
\left(1-q^{2 m}\right) B_{m}(q)=B_{m-1}(q)+q^{2 m-1} B_{m-r}(q) .
$$

Now in Theorem 2 replace $q$ by $q^{2}$, then set $a_{1}=a_{2}=\ldots=a_{r-1}=0, a_{r}=q^{-1}$. This yields

$$
\begin{aligned}
1+\sum_{n=1}^{\infty} P_{2}(n) q^{n} & =\lim _{m \rightarrow \infty} B_{m}(q) \\
& =\prod_{j=1}^{\infty}\left(1+q^{2 r j-1}\right)\left(1-q^{2 j}\right)^{-1} \\
& =\prod_{j=1}^{\infty}\left(1-q^{4 r j-2}\right)\left(1-q^{2 j}\right)^{-1}\left(1-q^{2 r q-1}\right)^{-1} \\
& =1+\sum_{n=1}^{\infty} P_{1}(n) q^{n} .
\end{aligned}
$$

Comparing coefficients on both sides of (3.12), we obtain Theorem 3 .

Theorem 4 (stated in the introduction) is obtained from Theorem 3 directly; set $r=2$ in Theorem 3.

4. Schur's recurrence lemma. The following theorem is a strengthened form of the result Schur originally used to prove Theorem 1 . We shall show that the result is a consequence of the $q$-analogue of Gauss's theorem for hypergeometric series [4, p. 97, (3.3.2.5)].

THEOREM 5. If $P_{0}=1$,

$$
P_{n}=\prod_{j=1}^{n}\left(1+\alpha q^{j}+z q^{2 j}\right),
$$


and $D_{n}$ is defined by $D_{0}=1, D_{1}=1+\alpha q$,

then

$$
D_{n}=\left(1+\alpha q^{n}\right) D_{n-1}+z q^{n}\left(1-q^{n-1}\right) D_{n-2} \quad(n>1) \text {, }
$$

where

$$
D_{n}=\sum_{m=0}^{n}(-z)^{m} q^{m(n+1)-\frac{1}{2} m(m-1)}\left[\begin{array}{l}
n \\
m
\end{array}\right] P_{n-m},
$$

$$
\left[\begin{array}{l}
n \\
m
\end{array}\right]=\prod_{j=1}^{m}\left(1-q^{n-j+1}\right)\left(1-q^{j}\right)^{-1} .
$$

Proof. Let $\beta_{1}$ and $\beta_{2}$ be the roots of the equation $x^{2}+\alpha x+z=0$. Then, by (3.3) and (3.4),

$$
\prod_{n=1}^{\infty}\left(1-\beta_{1} x q^{n}\right)\left(1-\beta_{2} x q^{n}\right)\left(1-x q^{n-1}\right)^{-1}=\sum_{n=0}^{\infty} D_{n} x^{n} \prod_{j=1}^{n}\left(1-q^{j}\right)^{-1}
$$

But, by the $q$-analogue of Gauss's theorem $[4$, p. 97, (3.3.2.5)],

$$
\begin{aligned}
\prod_{n=1}^{\infty} & \left(1-\beta_{1} x q^{n}\right)\left(1-\beta_{2} x q^{n}\right)\left(1-x q^{n-1}\right)^{-1} \\
& =\sum_{N=0}^{\infty} x^{N} \prod_{j=1}^{N}\left(1-\beta_{1} q^{j}\right)\left(1-\beta_{2} q^{j}\right)\left(1-q^{j}\right)^{-1} \prod_{k=0}^{\infty}\left(1-x z q^{h+N+2}\right) \\
& =\sum_{N=0}^{\infty} \sum_{k=0}^{\infty} P_{N} x^{N}(-1)^{k} z^{k} q^{(N+2) k+\frac{1}{2} k(k-1)} x^{k} \prod_{m=1}^{N}\left(1-q^{m}\right)^{-1} \prod_{j=1}^{k}\left(1-q^{j}\right)^{-1} \\
& =\sum_{n=0}^{\infty}\left(\sum_{N+k=n} P_{N}(-z)^{k} q^{(N+2) k+\frac{1}{2} k(k-1)} \prod_{m=1}^{N}\left(1-q^{m}\right)^{-1} \prod_{j=1}^{k}\left(1-q^{j}\right)^{-1}\right) x^{n},
\end{aligned}
$$

where the penultimate expression is obtained by expanding the infinite product in the sum and by applying Euler's theorem [4, p. 92, (3.2.2.15)].

Comparing coefficients of $x^{n}$ in the series expansion of (4.2) and (4.3), we obtain

$$
\begin{aligned}
D_{n} & =\sum_{N+k=n}(-z)^{k} P_{N} q^{(N+2) k+\frac{1}{2} k(k-1)}\left[\begin{array}{l}
n \\
k
\end{array}\right] \\
& =\sum_{k=0}^{n}(-z)^{k} q^{(n-k+2) k+\frac{1}{2} k(k-1)}\left[\begin{array}{l}
n \\
k
\end{array}\right] P_{n-k} \\
& =\sum_{k=0}^{n}(-z)^{k} q^{k(n+1)-\frac{1}{2} k(k-1)}\left[\begin{array}{l}
n \\
k
\end{array}\right] P_{n-k} .
\end{aligned}
$$

\section{REFERENCES}

1. P. Dienes, The Taylor series, Dover (New York, 1957).

2. W. Gleissberg, Über einen Satz von Herrn I. Schur, Math. Z. 28 (1928), 372-382.

3. I. J. Schur, Zur additiven Zahlentheorie, S.-B. Akad. Wiss. Berlin (1926), 488-495. 1966).

4. L. J. Slater, Generalized hypergeometric functions, Cambridge University Press (Cambridge,

The Pennsylvania State University

University Park, PenNSYlVania 